\title{
Surround Inhibition in the Primary Motor Cortex is Task-specifically Modulated in Non-professional Musicians but not in Healthy Controls During Real Piano Playing
}

\author{
Gonzalo Márquez, ${ }^{a *}$ Martin Keller, ${ }^{b}$ Jesper Lundbye-Jensen ${ }^{c}$ and Wolfgang Taube ${ }^{b}$ \\ ${ }^{a}$ Department of Physical Education and Sports, UCAM, Catholic University San Antonio, Murcia, Spain \\ ${ }^{\mathrm{b}}$ Movement and Sport Science, Department of Medicine, University of Fribourg, Fribourg, Switzerland \\ ${ }^{\mathrm{c}}$ Department of Nutrition, Exercise and Sports, University of Copenhagen, Copenhagen, Denmark
}

\begin{abstract}
Research has indicated that at the onset of a finger movement, unwanted contractions of adjacent muscles are prevented by inhibiting the cortical areas representing these muscles. This so-called surround inhibition (SI) seems relevant for the performance of selective finger movements but may not be necessary for tasks involving functional coupling between different finger muscles. Therefore, the present study compared SI between isolated finger movement and complex selective finger movements while playing a three-finger sequence on the piano in nine non-professional musicians and 10 untrained control participants. Transcranial magnetic stimulation (TMS) was applied to the contralateral motor cortex to assess SI in the first dorsal interosseous (FDI), abductor pollicis brevis (APB) and abductor digiti minimi (ADM) during the movement preparation and the late phasic phases. The results reveal stronger SI during the preparation phase than during the phasic phase $(30.6 \% \mathrm{vs}$. $10.7 \% ; P<0.05)$ in the isolated-finger condition in both musicians and controls. Results also show higher SI in musicians during the preparation phase of the isolated finger condition compared to the preparation phase of the three-finger sequence $(40 \%$ vs. $15 \% ; P<0.05)$. However, the control group did not show this taskspecific modulation of $\mathrm{SI}$ (isolated: $25 \%$ vs. sequence: $25 \% ; P>0.05$ ). Thus, musicians were able to modulate SI between conditions whereas control participants revealed constant levels of SI. Therefore, it may be assumed that long-term training as observed in skilled musicians is accompanied by task-specific effects on SI modulation potentially relating to the ability to perform selective and complex finger movements. (C) 2018 IBRO. Published by Elsevier Ltd. All rights reserved.
\end{abstract}

Key words: motor surround inhibition, transcranial magnetic stimulation, primary motor cortex, task complexity, musicians.

\section{INTRODUCTION}

Surround inhibition $(\mathrm{SI})$ is a mechanism to focus neuronal activity (Angelucci et al., 2002). It was originally detected in the sensory system (Blakemore et al., 1970), and later, $\mathrm{SI}$ has also been described in the primary motor cortex (M1) (Mink, 1996; Sohn and Hallett, 2004b). There is evidence that SI, mediated through GABAergic transmission, could relate to the ability to perform selective

${ }^{*}$ Corresponding author. Address: Facultad de Deporte - Universidad Católica de Murcia, Campus de los Jerónimos, s/n, 30107 Guadalupe, Murcia, Spain. Fax: +34-968-278-658.

E-mail address: gmarquez@ucam.edu (G. Márquez).

Abbreviations: ADM, abductor digiti minimi; APB, abductor pollicis brevis; bEMG, background EMG; EMG, electromyography; FDI, first dorsal interosseous; GABA, gamma-amino-butyric acid; ICF, intracortical facilitation; M1, primary motor cortex; MEP, motor-evoked potential; RMT, resting motor threshold; SD, standard deviation; SE, standard error; SI, surround inhibition; $\mathrm{SICl}$, short intra-cortical inhibition; TMS, transcranial magnetic stimulation. movements in humans (Mink, 1996; Ziemann et al., 1996a,b).

During and prior to contractions, corticospinal excitability is increased for circuits involved in activating the prime mover muscles, while corticospinal excitability is reduced for connections to neighboring muscles (Sohn and Hallett, 2004a,b). Studies using single-pulse transcranial magnetic stimulation (TMS) to evoke motorevoked potentials (MEPs) showed facilitated MEPs in the active muscle (agonist) before the onset of muscle activity and during the movement. In contrast, MEPs in surrounding and inactive muscles are inhibited before and directly after movement initiation (Sohn and Hallett, 2004b; Beck et al., 2008). While the time course of this MEP modulation in the active muscle (prime mover) seems to be independent of the type of task performed (simple reaction time task, choice reaction time task or go/no-go-task) (Leocani et al., 2000), the SI occurs earlier and is more pronounced in a choice reaction time task compared with a simple reaction time task (Beck and 
Hallett, 2010), and it is also related to the force exerted by the active muscle (Beck et al., 2009b). Thus, there is evidence that $\mathrm{SI}$ is modulated in a task- and phase-specific manner. From a functional point of view, it has been suggested that SI focuses neural activation to the prime mover and prevents unwanted overflow of activity to surrounding muscles. Shin et al., (2009) therefore tested SI in $\mathrm{M} 1$ for both hemispheres (i.e. for both the dominant and non-dominant upper limb). They found a hemispheric asymmetry of SI with a more prominent SI in the dominant hemisphere and argued that the increased SI for the dominant hand might be one factor contributing to the greater dexterity in the dominant limb. Thus, SI may be relevant for skilled motor performance and it may also be subject to use-dependent plasticity. The latter point is further supported by the observation that short-term motor training can transiently influence the amount of SI. For example, isolated index finger movements of the first dorsal interosseous (FDI) reduced MEPs in the surrounding muscles such as abductor pollicis brevis (APB) and/or the abductor digiti minimi (ADM) (Kassavetis et al., 2012; Sugawara et al., 2012). Furthermore, intracortical inhibition was shown to increase in the surrounding muscles after motor skill learning (Bütefisch et al., 2005; Sugawara et al., 2012). However, in contrast to the aforementioned studies demonstrating increased levels of SI during isolated finger movements, the training of synchronized finger movements was shown to reduce SI in surrounding muscles (Kang et al., 2012, 2013). In line with this, professional musicians who frequently train multidigit tasks that involve muscle synergies seem to have permanently reduced SI compared to healthy controls (Shin et al., 2012; Kang et al., 2013). Nordstrom and Butler (2002) have also found reduced short intracortical inhibition (SICl) and facilitation (ICF) of corticospinal neu- rons in musicians at rest and during weak voluntary contractions, supporting the idea of an altered balance between the excitatory and inhibitory inputs to corticospinal neurons.

The abovementioned observations imply that there is the need for further research clarifying the functional relevance of $\mathrm{SI}$ during selective and coupled finger movements. Therefore, the present study aimed to investigate the implications of $\mathrm{SI}$ during real piano playing, and how $\mathrm{SI}$ is affected by task complexity (isolated finger movement or a three-finger sequence) and previous experience (musicians vs. control participants). We hypothesized that musicians should illustrate use-dependent plasticity of SI that should not be evident in healthy participants. Furthermore, we expected to see a cohort-specific differential modulation of $\mathrm{SI}$ when switching from an isolated (single finger movement) to a coupled three-finger task (three finger movement). More specifically, we speculated that musicians are better able to task-specifically tune their $\mathrm{SI}$, thus, increase $\mathrm{SI}$ in the single finger task but decrease $\mathrm{SI}$ in the more complex three-finger task.

\section{EXPERIMENTAL PROCEDURES}

\section{Participants}

Nineteen healthy right-handed participants (10 untrained participants; age $27.9 \pm 4.3$ years, four females; and nine experienced non-professional musicians $30.7 \pm 8$. 8 years, four females) were enrolled in this study. Detailed information regarding the experience and primary instrument of our participants is shown in Table 1. Furthermore, all the musicians played their instrument only in leisure time and not more than $3 \mathrm{~h}$ per week during, at least, the preceding two years.

Table 1. Participants' description

\begin{tabular}{|c|c|c|c|c|c|c|c|c|c|}
\hline Group & Subject & $\begin{array}{l}\text { Age } \\
\text { (years) }\end{array}$ & $\begin{array}{l}\text { Weight } \\
(\mathrm{kg})\end{array}$ & $\begin{array}{l}\text { Height } \\
(\mathrm{m})\end{array}$ & $\begin{array}{l}\text { Handedness } \\
(R / L)\end{array}$ & $\begin{array}{l}\mathrm{RMT} \\
\text { (\% SO) }\end{array}$ & $\begin{array}{l}140 \% \\
\text { RMT }\end{array}$ & Instrument & $\begin{array}{l}\text { Experience } \\
\text { (years) }\end{array}$ \\
\hline \multirow[t]{9}{*}{ MUSICIANS } & 1 & 27 & 61 & 170 & $\mathrm{R}$ & 42 & 58 & PIANO & 23 \\
\hline & 2 & 41 & 105 & 184 & $\mathrm{R}$ & 52 & 73 & PIANO & 25 \\
\hline & 3 & 27 & 65 & 168 & $\mathrm{R}$ & 34 & 45 & PIANO & 8 \\
\hline & 4 & 37 & 82 & 187 & $\mathrm{R}$ & 30 & 40 & PIANO & 20 \\
\hline & 5 & 26 & 67 & 167 & $\mathrm{R}$ & 40 & 57 & GUITAR & 10 \\
\hline & 6 & 23 & 74 & 178 & $\mathrm{R}$ & 36 & 51 & SAXO & 15 \\
\hline & 7 & 22 & 65 & 178 & $\mathrm{R}$ & 38 & 53 & PIANO & 10 \\
\hline & 8 & 26 & 43 & 150 & $\mathrm{R}$ & 50 & 70 & PIANO & 16 \\
\hline & 9 & 47 & 59 & 180 & $\mathrm{R}$ & 38 & 55 & PIANO & 20 \\
\hline Average & & 30,7 & 69,0 & 173,6 & - & 40,0 & 55,8 & - & 16,3 \\
\hline \multirow[t]{10}{*}{ CONTROLS } & 1 & 37 & 90 & 185 & $\mathrm{R}$ & 67 & 85 & & \\
\hline & 2 & 28 & 70 & 182 & $\mathrm{R}$ & 42 & 58 & & \\
\hline & 3 & 30 & 87 & 186 & $\mathrm{R}$ & 47 & 65 & & \\
\hline & 4 & 31 & 78 & 170 & $\mathrm{R}$ & 35 & 49 & & \\
\hline & 5 & 24 & 75 & 177 & $\mathrm{R}$ & 46 & 64 & & \\
\hline & 6 & 29 & 70 & 181 & $\mathrm{R}$ & 30 & 42 & & \\
\hline & 7 & 22 & 70 & 180 & $\mathrm{R}$ & 37 & 52 & & \\
\hline & 8 & 24 & 63 & 172 & $\mathrm{R}$ & 33 & 46 & & \\
\hline & 9 & 28 & 68 & 168 & $\mathrm{R}$ & 31 & 43 & & \\
\hline & 10 & 26 & 58 & 158 & $\mathrm{R}$ & 38 & 53 & & \\
\hline Average & & 27,9 & 72,9 & 175,9 & - & 40,6 & 55,7 & - & - \\
\hline
\end{tabular}

R/L: right/left; RMT: resting motor threshold; \% SO: \% stimulator output 
However, they were required to show their skills with the instrument before the beginning of the experiment to verify their expertise. All participants gave written informed consent prior to participation in the experiment. The protocol was approved by the Ethics Committee of the canton of Fribourg and all experimental procedures conformed to the Declaration of Helsinki II standards.

\section{General experimental procedure}

Participants were asked to play the piano i.e. press piano keys with the right hand thumb, index and little finger in two different conditions: playing the piano rhythmically with one finger (isolated single finger movement using each of the three fingers) or in a sequence using three fingers (three finger movement). In order to assess SI, TMS was applied over the contralateral motor cortex using an intensity that elicited MEP in all tested muscles: FDI, APB, and ADM. SI was assessed in two different phases of the movement as a phase-specific modulation was reported previously (Sohn and Hallett, 2004b; Beck et al., 2008). Therefore, two different timepoints for stimulation were used in all conditions: TMS was elicited either during the movement preparation (50 ms before EMG onset) or directly after pressing the key of the piano (phasic phase). MEPs recorded at rest served as control MEPs and were defined as $100 \%$. Thus, all MEPs recorded in the isolated or three finger conditions were normalized to the rest condition. In the presence of SI, the normalized MEPs have values smaller than $100 \%$ of the control MEP measured at rest.

\section{Behavioral task}

During the experiment, participants sat in a comfortable position with their right forearm supported on a desk while the hand was placed with the fingertips of the thumb, index and little finger on three different keys of the piano (see Fig. 1A). The participants were asked to press the piano keys matching the sounds of a metronome that imposed a cadence of $80 \mathrm{bpm}$. In a first condition, participants pressed one key rhythmically with one of the three fingers while the other two fingers remained relaxed. In a second and third series of this condition, another finger hit the key while the other two fingers were at rest. Therefore, three different series were measured in this isolated finger condition (series1: FDI active with ADM and APB at rest; series 2: ADM active with $F D I$ and $A P B$ at rest; series 3: APB active with $A D M$ and FDI at rest). In the second condition, participants were asked to play a pre-defined sequence in which they pressed the thumb, index and little finger keys in a successive order. Like in the isolated finger condition, participants were asked to match the hits of the keys with the sound of the metronome (see Fig. 1B). The different measurements (isolated finger movement $_{\mathrm{FDI}}$, isolated finger movement $_{\mathrm{ADM}}$, isolated

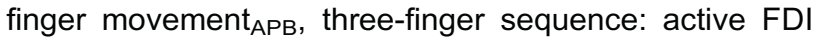
and active $A D M$ and active $A P B$ ) were run in a randomized order.

\section{Electromyography (EMG) recordings}

Surface EMG was recorded from the FDI, APB and ADM muscles of the right hand. Surface electrodes (Blue sensor, Ambu, Bad Nauheim, Germany) were attached to the skin in line with the presumed direction of the underlying muscle fibers in a bipolar electrode configuration (2 cm center-to-center distance). The reference electrode was placed on the ulnar styloid process. Electrodes and cables were secured with adhesive tape to prevent potential movement artifacts. EMG signals were amplified $(\times 1000)$, bandpass-filtered $(10-1000 \mathrm{~Hz})$ and sampled at $2 \mathrm{kHz}$ (LabView based

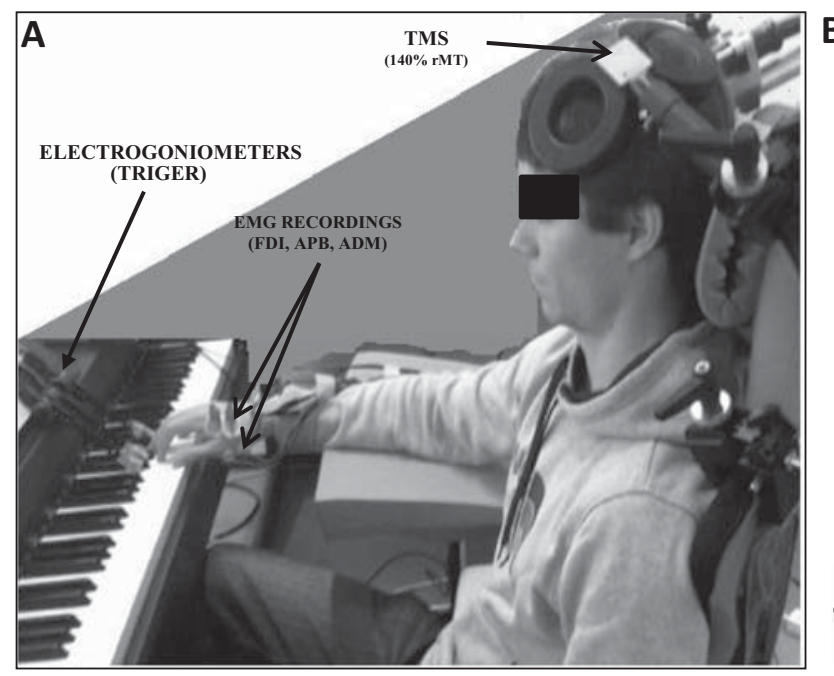

B
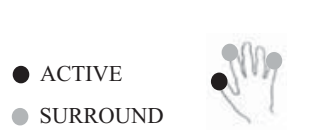

ISOLATED FINGER CONDITION

- SURROUND
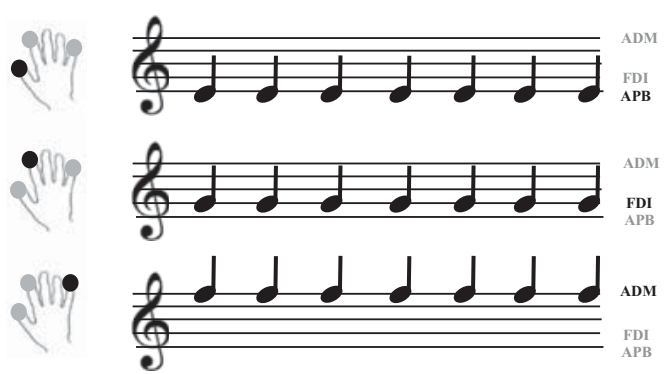

THREE FINGER CONDITION
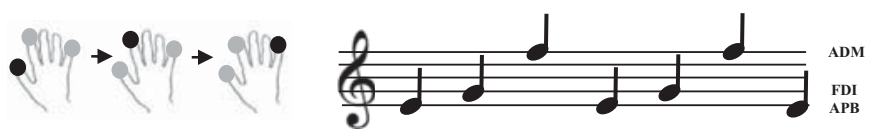

Fig. 1. Experimental set-up (A) and design (B). The left panel shows the experimental setup. One participant is playing the piano with the right hand. Transcranial magnetic stimulations are delivered to the left hemisphere. The design of the measurements is schematically displayed in the right panel. Surround inhibition was tested in an isolated and a three-finger condition. In both, the isolated and the three finger condition, motorevoked potentials and surround inhibition was assessed for three different muscles: abductor digiti minimi (ADM), first dorsal interosseous (FDI), and abductor pollicis brevis (APB). Whenever one muscle was active, surround inhibition was assessed in the other muscles (for example with active FDI: evaluation of surround inhibition in ADM and APB; etc). 
software; Pfitec Biomedical Systems, Endingen, Germany) for further off-line analysis.

\section{TMS}

Single-pulse TMS with a monophasic waveform was applied using a butterfly figure of eight coil (MagVenture D-B80) connected to a stimulator (MagPro X100 with Option, MagVenture A/S, Farum, Denmark). Magnetic stimulation was applied to the left $\mathrm{M} 1$ at the optimal position for eliciting MEPs in the FDI. The position of the coil was marked on the scalp with a felt pen to ensure proper coil placement throughout the experiment. Coil orientation was tangential to the scalp with the handle pointing backward and laterally at a $45^{\circ}$ angle to the sagittal plane in order to induce posterior-a nterior-directed current in the brain and to activate corticospinal neurons transsynaptically (Kaneko et al., 1996). Resting motor threshold (RMT) of the FDI muscle was defined as the stimulator intensity at which three of five consecutive MEPs reached $50 \mu \mathrm{V}$ (Kujirai et al., 1993). During measurements, the stimulation intensity was set to $140 \%$ RMT (Beck et al., 2008). This stimulation intensity ensured the activation of adjacent $M 1$ regions such as the representational areas of ADM and APB. This allowed the computation of MEPs and SI in the three muscles in all conditions. MEPs were recorded at rest and in two different time intervals (movement preparation and phasic phase) while participants played piano in both the single finger (active FDI and active ADM and active $A P B$ ) and the three finger conditions (active FDI and active $A D M$ and active APB).

As mentioned above, TMS was randomly applied during different phases of the movement. When stimulating during the phasic phase, TMS pulses were delivered immediately after pressing the desired key. Mechanical electrogoniometers were attached to the piano that triggered the stimulation. TMS pulses were triggered when the piano key was moved by approximately $5^{\circ}$ and therefore at the onset of the movement of each individual finger. When stimulating during the movement preparation phase, TMS was delivered approximately $50 \mathrm{~ms}$ prior to the onset of the initial EMG burst. The onset of the initial EMG burst was assessed in an adaptation phase without stimulation, and therefore, TMS was triggered by the precedent keystroke adjusting the time delay individually. An interstimulus interval of at least $5 \mathrm{~s}$ was chosen to minimize the effects of a preceding TMS stimulation on the size of the actual MEP. This interstimulus interval guaranteed that participants had sufficient time to retrieve the $80 \mathrm{bpm}$ pace of the metronome. In the single finger condition, TMS was always triggered by one specific key (e.g. the key pressed by the index finger when SI of ADM and APB was assessed). In the three-finger condition, TMS was randomly stimulated by one of the three different fingers. Thus, one among the three fingers randomly triggered TMS. This means that TMS could be triggered by the different keys (or fingers) but that the order of stimulation was randomly chosen by the computer program.

\section{Data analysis}

A total of 40 MEPs were recorded for the rest condition throughout the experimental session. Twenty MEPs were collected for each time interval, condition and active muscle (APB, FDI and ADM). Therefore, we have recorded 240 MEPs (20 MEPs x Muscle (3) x Condition (2) $x$ Phase (2)). Moreover, we also recorded 480 keystrokes without stimulation (EMG only).

MEPs were analyzed by evaluating peak-to-peak amplitudes for the different conditions (isolated vs. three-finger sequence) and time intervals (preparation and phasic phase). The level of background EMG (bEMG) was determined by calculating the root mean square (RMS) value in a 20 -ms time window from trials without stimulation. The level of bEMG was determined for the preparation and phasic phase to guarantee similar level of bEMG prior to stimulation.

\section{Statistical analysis}

A mixed RM-ANOVA design was performed with the factors TASK COMPLEXITY (single finger vs. three finger movement), PHASE (preparation vs. phasic), MUSCLE (FDI, APB and ADM) and GROUP (musicians vs. controls). This analysis was performed for the normalized MEP values. In order to test background EMG of the surround muscles, a three-way RM-ANOVA was performed with the factors SITUATION (rest, preparation-isolated, phasic-isolated, preparationsequence and phasic-sequence), MUSCLE (FDI, APB and ADM) and GROUP (musicians vs. controls). Kolmogorov-Smirnov test was used to test for normal distribution of the data and Levene test for homogeneity. The level for statistical significance was set at $P \leq 0.05$. Post-hoc analysis was performed using paired Student $t$-test comparisons with Bonferroni correction. SPSS 20.0 software (SPSS, Chicago, IL, USA) was used for statistical analysis. Data are presented as group mean values \pm standard error (SE) in figures and mean \pm standard deviation (SD) in tables.

\section{RESULTS}

SI: Regarding the level of SI, RM-ANOVA showed a main effect of PHASE $\left(F_{1,18}=15,124, P=0.001\right)$, a TASK COMPLEXITY*PHASE interaction $\left(F_{1},{ }_{18}=10.125\right.$, $P=0.005)$ and a TASK COMPLEXITY*PHASE ${ }^{*}$ GROUP interaction $\left(F_{1,18}=4.742, P=0.043\right)$. The post hoc comparisons revealed stronger SI during the preparation phase than in the phasic phase during the isolatedfinger condition in both musicians and controls (Musicians: $T=27.667, P=0.001$; Controls: $T=16.121$, $P=0.020$; Fig. 2). Results also show higher $\mathrm{SI}$ in musicians during the preparation phase of the isolated finger condition compared to the preparation phase of the three-finger sequence for all tested muscles $(P<0.05$ for all comparisons; Fig. 2). In contrast, the control group did not show an altered modulation of $\mathrm{SI}$ with a change in task complexity.

Muscular activity: The analysis of the bEMG for the surround muscles did not show any significant main 


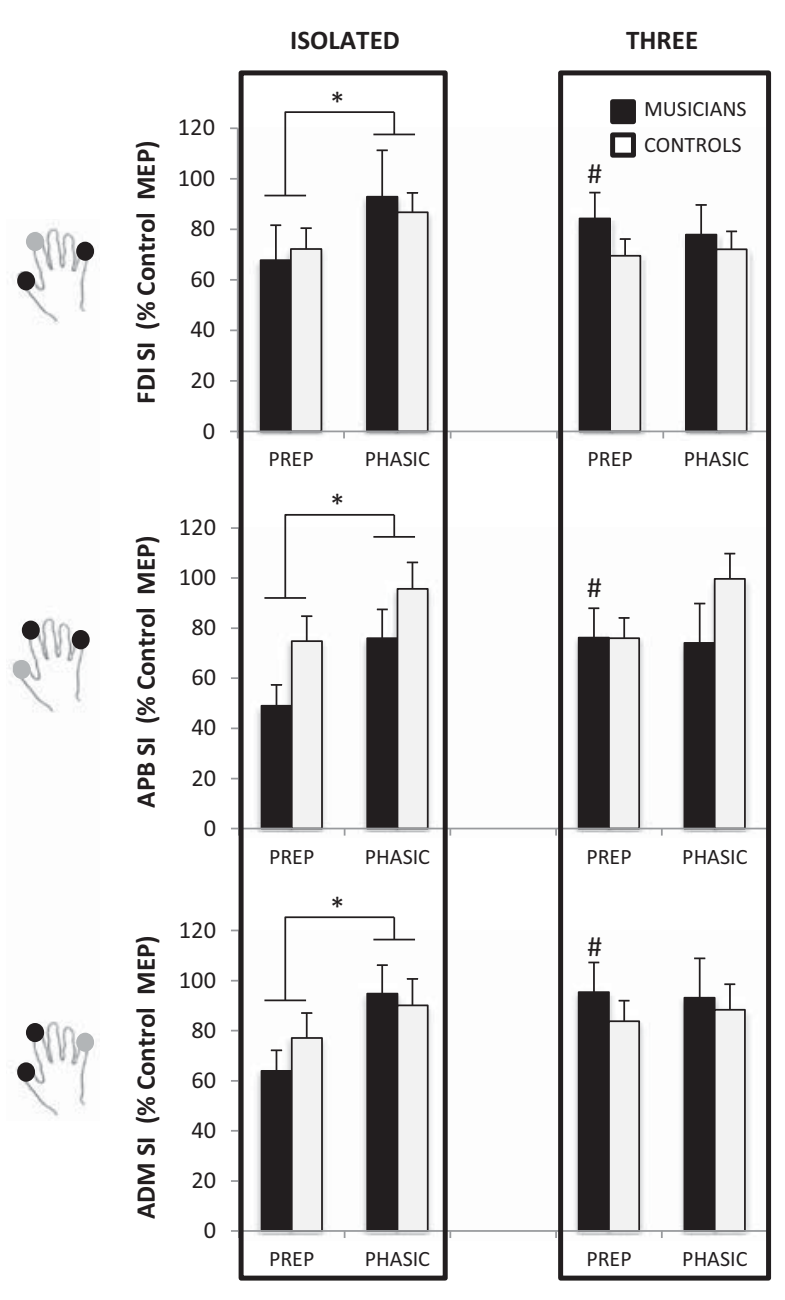

Fig. 2. Mean ( \pm SE) of the normalized motor-evoked potentials during the preparation and phasic phase of the isolated and threefinger sequence piano task. The black (active fingers) and grey (surround finger) circles in the hand indicates the role of each finger during the different conditions performed while participants played piano. Surround inhibition was, therefore, calculated as the average inhibition of the surround muscles while the other two muscles were active. Results revealed stronger surround inhibition (SI) in both groups during the preparation phase than in the phasic phase during the single finger condition in all tested muscles $\left({ }^{\star} P<0.05\right)$. Furthermore, higher levels of SI were found during the preparation phase of the single-finger condition compared to the three-finger condition for all tested muscles in musicians $\left({ }^{\#} P<0.05\right.$ compared to the musicians' $\mathrm{SI}$ during the preparation phase of the isolated finger condition). This effect was not seen in control participants (PREP: preparation phase; PHASIC: phasic phase; ADM: abductor digiti minimi; APB: abductor pollicis brevis; FRI: first dorsal interosseous).
(MUSCLE: $\quad F_{2,}, 34=1.391, \quad P=0.263 ; \quad$ SITUATION: $\left.F_{4}, \quad 68=0.383, \quad P=0.820\right)$ or interaction effect (MUSCLE ${ }^{*}$ SITUATION ${ }^{*}$ GROUP: $\quad F_{8}, \quad{ }_{136}=0.998$, $P=0.441$ ) indicating similar background EMG activity across the different conditions in both groups (see Table 2).

\section{DISCUSSION}

The results of the present study showed a phase- and task-specific modulation of $\mathrm{SI}$ that differed between musicians and control participants.

According to previous studies in healthy participants, SI was found for all tested muscles (Sohn and Hallett, 2004b; Beck et al., 2008, 2009a,b; Kassavetis et al., 2014). For the single finger condition, SI was more pronounced during the movement preparation phase compared to the phasic phase: during the preparation of the movement, SI was increased to $\sim 40 \%$ and $\sim 25 \%$ in non-professional musicians and controls, respectively. Much smaller SI was observed during the subsequent phasic phase (musicians $\sim 12 \%$ and controls $\sim 9 \%$ inhibition). This phase-specific modulation did not depend on different bEMG levels of the surrounding muscles as the bEMG in these adjacent muscles was similar across phases and conditions. Previous studies reported similar levels of SI during the preparation and the early phasic phase of the movement but a decrease in SI during the tonic phase (e.g. Beck et al., 2008; Beck et al., 2009a, b). The reason for this discrepancy may be related to the fact that stimulation in the phasic phase was relatively late in the present study. Stimulation took actually place when participants hit the piano key, therefore, it might be argued that the main task, i.e. the selective finger activation, was already fulfilled and the need for SI was decreasing. In our protocol, the TMS pulses were delivered $\sim 35 \mathrm{~ms}$ after the onset of EMG. This might have been too late as previous studies have shown that the amount of $\mathrm{SI}$ is reduced when TMS is applied later than $40 \mathrm{~ms}$ after the onset of muscle activity (Shin et al., 2012; Kang et al., 2012, 2013). Therefore, the need for SI to selectively inhibit the surrounding muscles might have already been fulfilled at this late point of time.

Interestingly and even more importantly, nonprofessional musicians modulated SI not only depending on the phase of the movement but also with respect to the complexity of the task: musicians demonstrated SI of around $40 \%$ during the preparation phase of the

Table 2. Mean $( \pm S D$ ) of background EMG of the surrounding muscles

\begin{tabular}{|c|c|c|c|c|c|c|}
\hline & & \multicolumn{3}{|l|}{ Isolated } & \multicolumn{2}{|l|}{ Three-finger } \\
\hline & & Rest & Preparation & Phasic & Preparation & Phasic \\
\hline \multirow[t]{2}{*}{ FDI (mV) } & MUS & $0.05 \pm 0.03$ & $0.07 \pm 0.04$ & $0.09 \pm 0.03$ & $0.09 \pm 0.06$ & $0.10 \pm 0.06$ \\
\hline & CTR & $0.07 \pm 0.07$ & $0.07 \pm 0.04$ & $0.10 \pm 0.05$ & $0.08 \pm 0.04$ & $0.08 \pm 0.04$ \\
\hline \multirow[t]{2}{*}{ APB $(m V)$} & MUS & $0.06 \pm 0.04$ & $0.08 \pm 0.06$ & $0.09 \pm 0.06$ & $0.05 \pm 0.03$ & $0.08 \pm 0.08$ \\
\hline & CTR & $0.07 \pm 0.07$ & $0.06 \pm 0.05$ & $0.08 \pm 0.06$ & $0.07 \pm 0.07$ & $0.12 \pm 0.09$ \\
\hline \multirow[t]{2}{*}{$\mathrm{ADM}(\mathrm{mV})$} & MUS & $0.06 \pm 0.09$ & $0.06 \pm 0.05$ & $0.05 \pm 0.04$ & $0.07 \pm 0.06$ & $0.03 \pm 0.02$ \\
\hline & CTR & $0.06 \pm 0.07$ & $0.09 \pm 0.07$ & $0.07 \pm 0.06$ & $0.10 \pm 0.10$ & $0.03 \pm 0.04$ \\
\hline
\end{tabular}

MUS: musicians; CTR: controls; mV: millivolts. 
isolated finger task, whereas a much lower inhibition $(15 \%)$ was found in the more complex three-finger sequence. In contrast, control participants did not display such a complexity-dependent modulation of SI and revealed $\mathrm{SI}$ levels of around $25 \%$ in both tasks. Therefore, it can be assumed that long-term training of finger movements enabled participants to better adapt their level of SI in a task-specific manner. The importance of task-specificity in the context of SI was previously demonstrated in studies where participants were asked to either train simple or more complex finger movements. When single (isolated) finger movements were exercised, SI and intracortical inhibition in the adjacent muscles was increased (Kassavetis et al., 2012; Sugawara et al., 2012) whereas training of synchronized finger movements was shown to reduce SI for the surrounding muscles (Kang et al., 2012). Thus, depending on the training task, SI was differently affected. Importantly, all these studies tested SI during simple finger movements. Thus, the current study is the first one to display differences in SI depending on the complexity of the test condition. The functional relevance of a task-specific adaptation of SI would be that isolated finger actions might depend on high SI whereas synergistic muscle movements could be fostered by decreasing SI. This observation may also nicely explain the decrease in SI when training synchronized (coupled) finger movements as this kind of training seems to strengthen inter-digit cortical connections and/or weaken inhibitory processes (Kang et al., 2013). Furthermore, in extreme cases such as high-level professional musicians, extensive training of coupled finger movements may lead to chronically decreased SI (Shin et al., 2012), probably making highlevel musicians more susceptible to develop focal hand dystonia (Stinear and Byblow, 2004).

When taking these aspects into consideration, the comparison of our well-trained but non-professional musicians with control participants (present study) and professional musicians (previous studies) indicates several important aspects with respect to the acquisition and modulation of SI: First, training/learning seems to alter not only SI but also to enable participants to better modulate $\mathrm{SI}$ between different conditions (comparison between the isolated and the three-finger task in the current study). Second, when functional coupling of finger muscles is needed, like in the three-finger sequence, SI can be task-specifically reduced. This seems especially relevant for conditions in which finger muscles act synchronously or as synergists. In line with this, Kang et al. (2012) demonstrated reduced SI after a synchronized finger exercise. Third, long-term extensive practice may impair the ability for task-specific SImodulation, probably leading to chronically reduced levels of SI. Another possible explanation is that attention may have played a significant role in this task-specific modulation of MEPs, and different levels of attentional demands between musicians and control participants may explain some of the differences in the modulation of SI. It might be argued that playing a sequence of notes, for a musician, is a much more natural and therefore less attention demanding task than for a non-musician, or even, it could change the attentional focus which has been shown to affect the excitatory-inhibitory balance of the intracortical circuitry of M1 (Kuhn et al., 2017).

\section{CONCLUSION}

The observation of a task-dependent modulation of $\mathrm{SI}$ is important to better understand the functional relevance of SI. It seems that when coupling of finger muscles is needed, $\mathrm{SI}$ is reduced whereas strengthening of $\mathrm{SI}$ occurs during isolated finger movements. The differential training outcomes reported in the literature may therefore most likely be explained by a taskdependent strengthening of this pattern during the training process. Furthermore, our population of wellexperienced but nevertheless non-professional musicians fills a gap in the scientific literature and helps to better understand changes and adaptations in SI. Compared to control participants, non-professional musicians demonstrated enhanced $\mathrm{SI}$ in isolated single finger movements - as one would expect based on the literature about short-term adaptations in SI after single finger movements. However, at the same time they displayed reduced $\mathrm{SI}$ in a complex task involving three fingers. This supports the assumption that $\mathrm{SI}$ is reduced when functional coupling of finger muscles is needed. Thus, non-professional musicians seem to have acquired a better ability to task-specifically modulate SI. In contrast, professional musicians are supposed to demonstrate chronically reduced $\mathrm{SI}$, which potentially may lead to pathological conditions when exaggerated. However, a limitation of this study is the small sample size. A previous study discussed the issue of variability of SI (Kassavetis et al., 2014). Therefore, the reported differences should be taken with caution due to the small sample size $(n=19)$. Further research is needed to shed more light on this specific topic with larger groups with different musical training background.

\section{ACKNOWLEDGMENTS}

GM was funded by the Spanish Ministry of Education, Culture and Sport (National Program for "Mobility stays abroad José Castillejo" for young doctors; Grant ref.: CAS14/00128), and by the Spanish Ministry of Economy and Competitiveness (Grant ref.: PSI2015-71061-P).

\section{REFERENCES}

Angelucci A, Levitt JB, Lund JS (2002) Anatomical origins of the classical receptive field and modulatory surround field of single neurons in macaque visual cortical area V1. In: Progress in brain research, vol. 136. p. 373-388. https://doi.org/10.1016/S00796123(02)36031-X.

Beck S, Hallett M (2010) Surround inhibition is modulated by task difficulty. Clin Neurophysiol 121(1):98-103. https://doi.org/ 10.1016/i.clinph.2009.09.010.

Beck S, Richardson SP, Shamim EA, Dang N, Schubert M, Hallett M (2008) Short intracortical and surround inhibition are selectively reduced during movement initiation in focal hand dystonia. $\mathrm{J}$ 
Neurosci 28(41):10363-10369. https://doi.org/10.1523/ JNEUROSCI.3564-08.2008.

Beck S, Houdayer E, Richardson SP, Hallett M (2009a) The role of inhibition from the left dorsal premotor cortex in right-sided focal hand dystonia. Brain Stimul 2(4):208-214. https://doi.org/ 10.1016/j.brs.2009.03.004.

Beck S, Schubert M, Richardson SP, Hallett M (2009b) Surround inhibition depends on the force exerted and is abnormal in focal hand dystonia. J Appl Physiol (Bethesda, Md.: 1985) 107 (5):1513-1518. https://doi.org/10.1152/iapplphysiol.91580.2008.

Blakemore C, Carpenter RH, Georgeson MA (1970) Lateral inhibition between orientation detectors in the human visual system. Nature 228:37-39. https://doi.org/10.1038/228037a0.

Bütefisch CM, Boroojerdi B, Chen R, Battaglia F, Hallett M (2005) Task-dependent intracortical inhibition is impaired in focal hand dystonia. Mov Disord 20(5):545-551.

Kaneko K, Kawai S, Fuchigami Y, Morita H, Ofuji A (1996) The effect of current direction induced by transcranial magnetic stimulation on the corticospinal excitability in human brain. Electroencephalogr Clin Neurophysiol 101(6):478-482. https:// doi.org/10.1016/S0921-884X(96)96021-X.

Kang SY, Hallett M, Sohn YH (2012) Synchronized finger exercise reduces surround inhibition. Clin Neurophysiol 123 (11):2227-2231. https://doi.org/10.1016/i.clinph.2012.04.019.

Kang SY, Hallett M, Sohn YH (2013) Exercise-induced strengthening of inter-digital connections in musicians. Clin Neurophysiol 124 (8):1622-1627. https://doi.org/10.1016/j.clinph.2013.01.028.

Kassavetis P, Saifee TA, Sadnicka A, Pareés I, Kojovic M, Rothwell JC, Edwards MJ (2012) Adaptation of surround inhibition in the human motor system. Exp Brain Res 222(3):211-217.

Kassavetis P, Sadnicka A, Saifee TA, Belvisi D, Van den Bos M, Pareés I, Ellipsis Edwards MJ (2014) Motor "surround inhibition" is not correlated with activity in surround muscles. Eur J Neurosci 40(3):2541-2547. https://doi.org/10.1111/ejn.12613.

Kuhn YA, Keller M, Ruffieux J, Taube W (2017) Adopting an external focus of attention alters intracortical inhibition within the primary motor cortex. Acta Physiol 220:289-299. https://doi.org/10.1111/ apha.12807.
Kujirai T, Caramia MD, Rothwell JC, Day BL, Thompson PD, Ferbert A (1993) Corticocortical inhibition in human motor cortex. J Physiol 471:501-519. https://doi.org/VL-471.

Leocani L, Cohen LG, Wassermann EM, Ikoma K, Hallett M (2000) Human corticospinal excitability evaluated with transcranial magnetic stimulation during different reaction time paradigms. Brain 123(6):1161-1173. https://doi.org/10.1093/brain/ 123.6.1161.

Mink JW (1996) The basal ganglia: focused selection and inhibition of competing motor programs. Prog Neurobiol. https://doi.org/ 10.1016/S0301-0082(96)00042-1.

Nordstrom MA, Butler SL (2002) Reduced intracortical inhibition and facilitation of corticospinal neurons in musicians. Exp Brain Res 144(3):336-342. https://doi.org/10.1007/s00221-002-1051-7.

Shin HW, Kang SY, Hallett M, Sohn YH (2012) Reduced surround inhibition in musicians. Exp Brain Res 219(3):403-408. https://doi. org/10.1007/s00221-012-3102-z.

Sohn YH, Hallett M (2004a) Disturbed surround inhibition in focal hand dystonia. Ann Neurol 56(4):595-599. https://doi.org/ 10.1002/ana.20270.

Sohn YH, Hallett M (2004b) Surround inhibition in human motor system. Exp Brain Res 158(4):397-404. https://doi.org/10.1007/ s00221-004-1909-y.

Stinear CM, Byblow WD (2004) Impaired modulation of intracortical inhibition in focal hand dystonia. Cereb Cortex (New York, NY: 1991) 14(5):555-561. https://doi.org/10.1093/cercor/bhh017.

Sugawara K, Tanabe S, Higashi T, Suzuki T, Tsurumi T, Kasai T (2012) Functional plasticity of surround inhibition in the motor cortex during single finger contraction training. NeuroReport 23 (11):663-667. https://doi.org/10.1097/WNR.0b013e3283556522.

Ziemann U, Lonnecker S, Steinhoff BJ, Paulus W (1996a) Effects of antiepileptic drugs on motor cortex excitability in humans: a transcranial magnetic stimulation study. Ann Neurol 40 (3):367-378. https://doi.org/10.1002/ana.410400306.

Ziemann U, Rothwell JC, Ridding MC (1996b) Interaction between intracortical inhibition and facilitation in human motor cortex. $J$ Physiol 496(Pt 3):873-881. https://doi.org/10.1113/jphysiol.1996. sp021734. 